\title{
REPRODUCING KERNEL FOR A CLASS OF WEIGHTED BERGMAN SPACES ON THE SYMMETRIZED POLYDISC
}

\author{
GADADHAR MISRA, SUBRATA SHYAM ROY, AND GENKAI ZHANG \\ (Communicated by Richard Rochberg)
}

\begin{abstract}
A natural class of weighted Bergman spaces on the symmetrized polydisc is isometrically embedded as a subspace in the corresponding weighted Bergman space on the polydisc. We find an orthonormal basis for this subspace. It enables us to compute the kernel function for the weighted Bergman spaces on the symmetrized polydisc using the explicit nature of our embedding. This family of kernel functions includes the Szegö and the Bergman kernel on the symmetrized polydisc.
\end{abstract}

\section{INTRODUCTION}

Let $\varphi_{i}, i \geq 0$, be the elementary symmetric function of degree $i$; that is, $\varphi_{i}$ is the sum of all products of $i$ distinct variables $z_{i}$ so that $\varphi_{0}=1$ and

$$
\varphi_{i}\left(z_{1}, \ldots, z_{n}\right)=\sum_{1 \leq k_{1}<k_{2}<\ldots<k_{i} \leq n} z_{k_{1}} \cdots z_{k_{i}} .
$$

For a fixed $n \geq 1$, let $\mathbf{s}: \mathbb{C}^{n} \longrightarrow \mathbb{C}^{n}$ be the function of symmetrization given by the formula

$$
\mathbf{s}\left(z_{1}, \ldots, z_{n}\right)=\left(\varphi_{1}\left(z_{1}, \ldots, z_{n}\right), \ldots, \varphi_{n}\left(z_{1}, \ldots, z_{n}\right)\right) .
$$

The image $\mathbb{G}_{n}:=\mathbf{s}\left(\mathbb{D}^{n}\right)$ under the map $\mathbf{s}$ of the unit polydisc $\mathbb{D}^{n}:=\left\{\boldsymbol{z} \in \mathbb{C}^{n}\right.$ : $\left.\|\boldsymbol{z}\|_{\infty}<1\right\}$ is known as the symmetrized polydisc. The restriction $\mathbf{s}_{\mid \mathrm{res}} \mathbb{D}^{n}: \mathbb{D}^{n} \rightarrow \mathbb{G}_{n}$ of the map $\mathbf{s}$ to $\mathbb{D}^{n}$ is a proper holomorphic map [9]. The Bergman kernel for the symmetrized polydisc is computed explicitly in [7]. It is obtained from the transformation rule for the Bergman kernel under proper holomorphic maps [3, Theorem 1].

Here we realize (isometrically) the Bergman space $\mathbb{A}^{2}\left(\mathbb{G}_{n}\right)$ of the symmetrized polydisc as a subspace of the Bergman space $\mathbb{A}^{2}\left(\mathbb{D}^{n}\right)$ on the polydisc using the symmetrization map s. Indeed, the map $\Gamma: \mathbb{A}^{2}\left(\mathbb{G}_{n}\right) \rightarrow \mathbb{A}^{2}\left(\mathbb{D}^{n}\right)$ defined by the formula

$$
(\Gamma f)(\boldsymbol{z})=(f \circ \mathbf{s})(\boldsymbol{z}) J_{\mathbf{s}}(\boldsymbol{z}), \boldsymbol{z} \in \mathbb{D}^{n}
$$

Received by the editors June 20, 2011 and, in revised form, October 17, 2011.

2010 Mathematics Subject Classification. Primary 30H20, 46E22, 47 B32.

Key words and phrases. Symmetrized polydisc, permutation group, sign representation, Schur functions, weighted Bergman space, Hardy space, weighted Bergman kernel, Szegö kernel.

Financial support for the work of the first and third authors was provided by the Swedish Research Links programme entitled "Hilbert modules, operator theory and complex analysis". The research of the first and second authors was funded by grants from DST and UKIERI. 
where $J_{\mathbf{s}}$ is the complex Jacobian of the map $\mathbf{s}$, is an isometric embedding. The functions in the image $\operatorname{ran} \Gamma \subseteq \mathbb{A}^{2}\left(\mathbb{D}^{n}\right)$ are anti-symmetric:

$$
\operatorname{ran} \Gamma:=\left\{f: f\left(\boldsymbol{z}_{\sigma}\right)=\operatorname{sgn}(\sigma) f(\boldsymbol{z}), \sigma \in \Sigma_{n}, f \in \mathbb{A}^{2}\left(\mathbb{D}^{n}\right)\right\},
$$

where $\Sigma_{n}$ is the symmetric group on $n$ symbols. The image of $\Gamma$ coincides with $\mathbb{A}_{\text {anti }}^{2}\left(\mathbb{D}^{n}\right)$, the subspace of anti-symmetric functions in $\mathbb{A}^{2}\left(\mathbb{D}^{n}\right)$. An orthonormal basis of $\mathbb{A}_{\text {anti }}^{2}\left(\mathbb{D}^{n}\right)$ may then be transformed into an orthonormal basis of the $\mathbb{A}^{2}\left(\mathbb{G}_{n}\right)$ via the unitary map $\Gamma^{*}$. It is then possible to compute the Bergman kernel for the symmetrized polydisc $\mathbb{G}_{n}$ by evaluating the sum

$$
\sum_{k \geq 0} e_{k}(\boldsymbol{z}) \overline{e_{k}(\boldsymbol{w})}, \boldsymbol{z}, \boldsymbol{w} \in \mathbb{G}_{n},
$$

for some choice of an orthonormal basis in $\mathbb{A}^{2}\left(\mathbb{G}_{n}\right)$.

This scheme works equally well for a class of weighted Bergman spaces $\mathbb{A}^{(\lambda)}\left(\mathbb{D}^{n}\right)$, $\lambda>1$, determined by the kernel function

$$
\mathbf{B}_{\mathbb{D}^{n}}^{(\lambda)}(\boldsymbol{z}, \boldsymbol{w})=\prod_{i=1}^{n}\left(1-z_{i} \bar{w}_{i}\right)^{-\lambda}, \boldsymbol{z}=\left(z_{1}, \ldots, z_{n}\right), \boldsymbol{w}=\left(w_{1}, \ldots, w_{n}\right) \in \mathbb{D}^{n},
$$

defined on the polydisc and the corresponding weighted Bergman spaces $\mathbb{A}^{(\lambda)}\left(\mathbb{G}^{n}\right)$ on the symmetrized polydisc.

The limiting case of $\lambda=1$, as is well-known, is the Hardy space on the polydisc. We show that the reproducing kernel for the Hardy space of the symmetrized polydisc is of the form

$$
\mathbb{S}_{\mathbb{G}_{n}}(\mathbf{s}(\boldsymbol{z}), \mathbf{s}(\boldsymbol{w}))=\prod_{i, j=1}^{n}\left(1-z_{i} \bar{w}_{j}\right)^{-1}, \boldsymbol{z}, \boldsymbol{w} \in \mathbb{D}^{n} .
$$

This is a consequence of the determinantal identity [8, (4.3), p. 63]. Indeed, along the way, we obtain a generalization of this well-known identity. We also point out that the Hardy kernel is not a power of the Bergman kernel unlike the case of bounded symmetric domains.

\section{Weighted Bergman spaces on the symmetrized PolydisC}

For $\lambda>1$, let $d V^{(\lambda)}$ be the probability measure $\left(\frac{\lambda-1}{\pi}\right)^{n}\left(\prod_{i=1}^{n}\left(1-r_{i}^{2}\right)^{\lambda-2} r_{i} d r_{i} d \theta_{i}\right)$ on the polydisc $\mathbb{D}^{n}$. Let $d V_{\mathbf{s}}^{(\lambda)}$ be the measure on the symmetrized polydisc $\mathbb{G}_{n}$ obtained by the change of variable formula. Throughout this note, by a suitable renormalization, we ignore the constant $n$ ! that would have otherwise appeared on the left-hand side of the equality below (cf. [2, p. 160]):

$$
\int_{\mathbb{G}_{n}} f d V_{\mathbf{s}}^{(\lambda)}=\int_{\mathbb{D}^{n}}(f \circ \mathbf{s})\left|J_{\mathbf{s}}\right|^{2} d V^{(\lambda)}, \lambda>1,
$$

where $J_{\mathbf{s}}(\boldsymbol{z})=\prod_{1 \leq i<j \leq n}\left(z_{i}-z_{j}\right)$ is the complex Jacobian of the symmetrization map s. Let $\left\|J_{\mathbf{s}}\right\|_{\lambda}^{2}=\int_{\mathbb{D}^{n}}\left|J_{\mathbf{s}}\right|^{2} d V^{(\lambda)}$ be the the norm of the Jacobian determinant $J_{\mathbf{s}}$ in the Hilbert space $L^{2}\left(\mathbb{D}^{n}, d V^{(\lambda)}\right)$. By a slight abuse of notation, we let $d V_{\mathbf{s}}^{(\lambda)}$ be the measure $\left\|J_{s}\right\|_{\lambda}^{-2} d V_{\mathbf{s}}^{(\lambda)}, \lambda>1$, on the symmetrized polydisc $\mathbb{G}_{n}$. The weighted Bergman space $\mathbb{A}^{(\lambda)}\left(\mathbb{G}_{n}\right), \lambda>1$, on the symmetrized polydisc $\mathbb{G}_{n}$ is the subspace of the Hilbert space $L^{2}\left(\mathbb{G}_{n}, d V_{\mathbf{s}}^{(\lambda)}\right)$ consisting of holomorphic functions. It coincides with the usual Bergman space for $\lambda=2$. The norm of $f \in \mathbb{A}^{(\lambda)}\left(\mathbb{G}_{n}\right)$ is given by 
$\|f\|^{2}=\int_{\mathbb{G}_{n}}|f|^{2} d V_{\mathbf{s}}^{(\lambda)}$. We have normalized the volume measure on $\mathbb{G}_{n}$ to ensure $\|1\|=1$. For $\lambda>1$, let $\Gamma: \mathbb{A}^{(\lambda)}\left(\mathbb{G}_{n}\right) \longrightarrow \mathbb{A}^{(\lambda)}\left(\mathbb{D}^{n}\right)$ be the operator defined by the rule:

$$
(\Gamma f)(\boldsymbol{z})=\left\|J_{\mathbf{s}}\right\|_{\lambda}^{-1} J_{\mathbf{s}}(\boldsymbol{z})(f \circ \mathbf{s})(\boldsymbol{z}), \quad f \in \mathbb{A}^{(\lambda)}\left(\mathbb{G}_{n}\right), \boldsymbol{z} \in \mathbb{D}^{n} .
$$

It is clear from the definition of the norm in $\mathbb{A}^{(\lambda)}\left(\mathbb{G}_{n}\right)$ that $\Gamma$ is an isometry.

Since $J_{\mathbf{s}}\left(\boldsymbol{z}_{\sigma}\right)=\operatorname{sgn}(\sigma) J_{\mathbf{s}}(\boldsymbol{z}), \sigma \in \Sigma_{n}$, it follows that the image $\Gamma\left(\mathbb{A}^{(\lambda)}\left(\mathbb{G}_{n}\right)\right)$ is contained in the subspace $\mathbb{A}_{\text {anti }}^{(\lambda)}\left(\mathbb{D}^{n}\right)$ of $\mathbb{A}^{(\lambda)}\left(\mathbb{D}^{n}\right)$ consisting of anti-symmetric functions. Thus ran $\Gamma \subseteq \mathbb{A}_{\text {anti }}^{(\lambda)}\left(\mathbb{D}^{n}\right)$. On the other hand, every function $g$ in $\mathbb{A}_{\text {anti }}^{(\lambda)}\left(\mathbb{D}^{n}\right)$ being anti-symmetric admits a factorization of the form $g(\boldsymbol{z})=J_{\mathbf{s}}(\boldsymbol{z}) h(\boldsymbol{z})$ for some holomorphic function $h$ on $\mathbb{D}^{n}$, which is symmetric. Therefore, the function $J_{\mathbf{s}}^{-1} g$ is well defined on the open set $\left\{\left(z_{1}, \ldots, z_{n}\right) \in \mathbb{D}^{n}: z_{i} \neq z_{j}, i \neq j\right\}$, where it agrees with $h$. Hence it extends to all of $\mathbb{D}^{n}$. This function $h=J_{\mathbf{s}}^{-1} g$ is symmetric and holomorphic. Consequently, it is of the form $f \circ \mathbf{s}$ for some function $f$ defined on $\mathbb{G}_{n}$. Therefore, the range of the isometry $\Gamma$ coincides with the subspace $\mathbb{A}_{\text {anti }}^{(\lambda)}\left(\mathbb{D}^{n}\right)$.

Now, it is easily verified that $\Gamma^{*} g=\left\|J_{\mathbf{s}}\right\|_{\lambda} f$, where $f$ is chosen satisfying $g(\boldsymbol{z})=$ $J_{\mathbf{s}}(\boldsymbol{z})(f \circ \mathbf{s})(\boldsymbol{z})$. The operator $\Gamma: \mathbb{A}^{(\lambda)}\left(\mathbb{G}_{n}\right) \longrightarrow \mathbb{A}_{\text {anti }}^{(\lambda)}\left(\mathbb{D}^{n}\right)$ is evidently unitary. The Hilbert spaces $\mathbb{A}^{(\lambda)}\left(\mathbb{G}_{n}\right), \lambda>1$, are the weighted Bergman spaces on the symmetrized polydisc $\mathbb{G}_{n}$.

Since the subspace $\mathbb{A}_{\text {anti }}^{(\lambda)}\left(\mathbb{D}^{n}\right)$ is invariant under the multiplication by the elementary symmetric function $\varphi_{i}, 1 \leq i \leq n$, we see that it admits a module action via the map

$$
(p, f) \mapsto p\left(\varphi_{1}, \ldots, \varphi_{n}\right) f, f \in \mathbb{A}_{\text {anti }}^{(\lambda)}\left(\mathbb{D}^{n}\right), p \in \mathbb{C}[\boldsymbol{z}]
$$

over the polynomial ring $\mathbb{C}[\boldsymbol{z}]$. Transplanting this action to the Hilbert space $\mathbb{A}^{(\lambda)}\left(\mathbb{G}_{n}\right)$ via $\Gamma$,

$$
(p, f) \mapsto p \cdot f:=\Gamma^{*}(p \cdot(\Gamma f)), f \in \mathbb{A}_{\text {anti }}^{(\lambda)}\left(\mathbb{G}_{n}\right),
$$

where $p$ is a polynomial in the symmetric variables $\left(\varphi_{1}(\boldsymbol{z}), \ldots, \varphi_{n}(\boldsymbol{z})\right)$, makes it a module over the symmetric polynomials. Indeed, $p \cdot f$ is the point-wise product of the symmetric polynomial $p$ and the function $f \in \mathbb{A}^{(\lambda)}\left(\mathbb{G}_{n}\right)$. Also, it is easily verified that the unitary operator $\Gamma$ intertwines the multiplication by the elementary symmetric functions on the Hilbert space $\mathbb{A}_{\text {anti }}^{(\lambda)}\left(\mathbb{D}^{n}\right)$ with the multiplication by the coordinate functions on $\mathbb{A}^{(\lambda)}\left(\mathbb{G}_{n}\right)$. Thus $\mathbb{A}^{(\lambda)}\left(\mathbb{G}_{n}\right)$ and $\mathbb{A}_{\text {anti }}^{(\lambda)}\left(\mathbb{D}^{n}\right)$ are isomorphic as modules via the unitary map $\Gamma$. Moreover, since $\mathbb{A}^{(\lambda)}\left(\mathbb{G}_{n}\right)$ is a submodule of the $L^{2}\left(\mathbb{G}_{n}, d V_{\mathbf{s}}^{(\lambda)}\right)$, it follows that the map

$$
(p, f) \mapsto p \cdot f, f \in \mathbb{A}^{(\lambda)}\left(\mathbb{G}_{n}\right), p \in \mathbb{C}[\boldsymbol{z}]
$$

is contractive. It therefore extends to a continuous map of the function algebra $\mathcal{A}\left(\mathbb{G}_{n}\right)$ obtained by taking the completion of the symmetric polynomials with respect to the supremum norm on the symmetrized polydisc $\mathbb{G}_{n}$. The reader may consult [5] for more details on Hilbert modules.

2.1. Orthonormal basis and kernel function. A partition $\mathbf{p}$ is any finite sequence $\boldsymbol{p}:=\left(p_{1}, \ldots, p_{n}\right)$ of nonnegative integers in decreasing order, that is,

$$
p_{1} \geq \cdots \geq p_{n} .
$$


We let $[n]$ denote the set of all partitions of size $n$. If a partition $\boldsymbol{p}$ also has the property $p_{1}>p_{2}>\cdots>p_{n} \geq 0$, then we may write $\boldsymbol{p}=\boldsymbol{m}+\boldsymbol{\delta}$, where $\boldsymbol{m}$ is some partition in $[n]$ and $\boldsymbol{\delta}=(n-1, n-2, \ldots, 1,0)$. Let $\llbracket n \rrbracket$ be the set of all partitions of the form $\boldsymbol{m}+\boldsymbol{\delta}$ for $\boldsymbol{m} \in[n]$.

Let $\boldsymbol{z}^{\boldsymbol{m}}:=z_{1}^{m_{1}} \cdots z_{n}^{m_{n}}, \boldsymbol{m} \in[n]$, be a monomial. Consider the polynomial $a_{\boldsymbol{m}}$ obtained by anti-symmetrizing the monomial $z^{m}$ :

$$
a_{\boldsymbol{m}}(\boldsymbol{z}):=\sum_{\sigma \in \sum_{n}} \operatorname{sgn}(\sigma) \boldsymbol{z}^{\boldsymbol{m}_{\sigma}}
$$

where $\boldsymbol{z}^{\boldsymbol{m}_{\sigma}}=z_{1}^{m_{\sigma(1)}} \cdots z_{n}^{m_{\sigma(n)}}$. Thus for any $\boldsymbol{p} \in \llbracket n \rrbracket$, we have

$$
a_{\boldsymbol{p}}(\boldsymbol{z})=a_{\boldsymbol{m}+\boldsymbol{\delta}}(\boldsymbol{z})=\sum_{\sigma \in \sum_{n}} \operatorname{sgn}(\sigma) \boldsymbol{z}^{(\boldsymbol{m}+\boldsymbol{\delta})_{\sigma}},
$$

$\boldsymbol{m} \in[n]$, and it follows that

$$
a_{\boldsymbol{p}}(\boldsymbol{z})=a_{\boldsymbol{m}+\boldsymbol{\delta}}(\boldsymbol{z})=\operatorname{det}\left(\left(\left(z_{i}^{p_{j}}\right)\right)_{i, j=1}^{n}\right), \boldsymbol{p} \in \llbracket n \rrbracket .
$$

The following lemma clearly shows that the functions $a_{\boldsymbol{p}}, \boldsymbol{p} \in \llbracket n \rrbracket$, are orthogonal in the Hilbert space $\mathbb{A}^{(\lambda)}\left(\mathbb{D}^{n}\right)$.

Lemma 2.1. Let $\boldsymbol{m}$ and $\boldsymbol{m}^{\prime}$ be two partitions in $\llbracket n \rrbracket$, that is, $m_{i}>m_{j}, m_{i}^{\prime}>m_{j}^{\prime}$ for all $i<j$. Assume that $m_{1}>m_{1}^{\prime}$ and fix $\sigma, \nu \in \Sigma_{n}$. Then the set $S:=$ $\left\{m_{\sigma(k)}-m_{\nu(k)}^{\prime}: 1 \leq k \leq n\right\} \neq\{0\}$.

Proof. If $\sigma(k)=\nu(k)=1$ for some $k, 1 \leq k \leq n$, then $m_{\sigma(k)}-m_{\nu(k)}^{\prime}=m_{1}-m_{1}^{\prime} \neq 0$. Therefore, in this case, $S \neq\{0\}$.

Now, suppose that there exists no $k, 1 \leq k \leq n$, for which $\sigma(k)=\nu(k)=1$. In this case, if possible, let $S=\{0\}$. Then there exists $k$ such that $\sigma(k)=1$ and $\nu(k)=j>1$. Now, $m_{\sigma(k)}-m_{\nu(k)}^{\prime}=m_{1}-m_{j}^{\prime}$. Pick $k^{\prime} \neq k$ such that $\sigma\left(k^{\prime}\right)=$ $j, \nu\left(k^{\prime}\right)=\ell, \ell \neq j$. Thus $m_{\sigma\left(k^{\prime}\right)}-m_{\nu\left(k^{\prime}\right)}^{\prime}=m_{j}-m_{\ell}^{\prime}$. Choose $k^{\prime \prime} \neq k$ such that $\nu\left(k^{\prime \prime}\right)=1, \sigma\left(k^{\prime \prime}\right)=r>1$ and $m_{\sigma\left(k^{\prime \prime}\right)}-m_{\nu\left(k^{\prime \prime}\right)}=m_{r}-m_{1}^{\prime}$. However, we have $m_{1}-m_{j}^{\prime}=m_{j}-m_{\ell}^{\prime}=m_{r}-m_{1}^{\prime}=0$. Clearly, $m_{r}=m_{1}^{\prime}>m_{j}^{\prime}=m_{1}$. Hence $m_{r}>m_{1}$ with $r>1$, which is a contradiction.

Since the vectors $\boldsymbol{z}^{\boldsymbol{p}_{\sigma}}$ are orthogonal in $\mathbb{A}^{(\lambda)}\left(\mathbb{D}^{n}\right)$, using Lemma 2.1. we see that the anti-symmetric polynomials $\left\{a_{\boldsymbol{p}}: \boldsymbol{p} \in \llbracket n \rrbracket\right\}$ are mutually orthogonal in it. Any anti-symmetric polynomial $p(z)$ can be written as $p(z)=a_{\boldsymbol{\delta}}(z) q(z)$, where $q$ is symmetric. But the Schur polynomials $\frac{a_{m+\delta}}{a_{\delta}}$ form a linear basis in the space of all symmetric polynomials [8, I, $(3,3)]$. In other words, $\boldsymbol{a}_{\boldsymbol{m}+\boldsymbol{\delta}}$ form a linear basis of all anti-symmetric polynomials. The anti-symmetric polynomials are dense in the Hilbert space $\mathbb{A}_{\text {anti }}^{(\lambda)}\left(\mathbb{D}^{n}\right)$. Therefore, the linear span of $\left\{a_{\boldsymbol{p}}: \boldsymbol{p} \in \llbracket n \rrbracket\right\}$ is dense in the Hilbert space $\mathbb{A}_{\text {anti }}^{(\lambda)}\left(\mathbb{D}^{n}\right)$.

For $\boldsymbol{p}=\left(p_{1}, \ldots, p_{n}\right) \in \llbracket n \rrbracket$, the norm of the vector $a_{\boldsymbol{p}}$ is easily calculated:

$$
\begin{aligned}
\left\|a_{\boldsymbol{p}}\right\|_{\mathbb{A}^{(\lambda)}\left(\mathbb{D}^{n}\right)} & =\left\|\operatorname{det}\left(\left(\left(z_{i}^{p_{j}}\right)\right)_{i, j=1}^{n}\right)\right\|_{\mathbb{A}^{(\lambda)}\left(\mathbb{D}^{n}\right)} \\
& =\left\|\sum_{\sigma \in \Sigma_{n}} \operatorname{sgn}(\sigma) \prod_{k=1}^{n} z_{k}^{p_{\sigma(k)}}\right\|_{\mathbb{A}^{(\lambda)}\left(\mathbb{D}^{n}\right)}=\sqrt{\frac{n ! \boldsymbol{p} !}{(\lambda)_{\boldsymbol{p}}}},
\end{aligned}
$$


where $\boldsymbol{p} !=\prod_{j=1}^{n} p_{j} !$ and $(\lambda)_{\boldsymbol{p}}=\prod_{j=1}^{n}(\lambda)_{p_{j}}$. Here $(\lambda)_{p_{j}}$ is the Pochhammer symbol $\lambda(\lambda+1) \cdots\left(\lambda+m_{j}-1\right)$. Putting $c_{\boldsymbol{p}}=\sqrt{\frac{(\lambda)_{p}}{n ! p !}}$, we see that

$$
\left\{e_{\boldsymbol{p}}=c_{\boldsymbol{p}} a_{\boldsymbol{p}}: \boldsymbol{p} \in \llbracket n \rrbracket\right\}
$$

is an orthonormal basis for $\mathbb{A}_{\text {anti }}^{(\lambda)}\left(\mathbb{D}^{n}\right)$. So the reproducing kernel $K_{\text {anti }}^{(\lambda)}$ for $\mathbb{A}_{\text {anti }}^{(\lambda)}\left(\mathbb{D}^{n}\right)$ is given by

$$
K_{\mathrm{anti}}^{(\lambda)}(\boldsymbol{z}, \boldsymbol{w})=\sum_{\boldsymbol{p} \in \llbracket n \rrbracket} e_{\boldsymbol{p}}(\boldsymbol{z}) \overline{e_{\boldsymbol{p}}(\boldsymbol{w})}, \text { for } \boldsymbol{z}, \boldsymbol{w} \in \mathbb{D}^{n} .
$$

For all $\sigma \in \Sigma_{n}$, we have $e_{\boldsymbol{p}_{\sigma}}(\boldsymbol{z}) \overline{e_{\boldsymbol{p}_{\sigma}}(\boldsymbol{w})}=e_{\boldsymbol{p}}(\boldsymbol{z}) \overline{e_{\boldsymbol{p}}(\boldsymbol{w})}, \boldsymbol{z}, \boldsymbol{w} \in \mathbb{D}^{n}$. Therefore, it follows that

$$
K_{\mathrm{anti}}^{(\lambda)}(\boldsymbol{z}, \boldsymbol{w})=\sum_{\boldsymbol{p} \in \llbracket n \rrbracket} e_{\boldsymbol{p}}(\boldsymbol{z}) \overline{e_{\boldsymbol{p}}(\boldsymbol{w})}=\frac{1}{n !} \sum_{\boldsymbol{p} \geq 0} e_{\boldsymbol{p}}(\boldsymbol{z}) \overline{e_{\boldsymbol{p}}(\boldsymbol{w})}
$$

where $\boldsymbol{p} \geq 0$ stands for all multi-indices $\boldsymbol{p}=\left(p_{1}, \ldots, p_{n}\right) \in \mathbb{Z}^{n}$ with the property that each $p_{i} \geq 0$ for $1 \leq i \leq n$.

Proposition 2.2. The reproducing kernel $K_{\mathrm{anti}}^{(\lambda)}$ is given explicitly by the formula

$$
K_{\text {anti }}^{(\lambda)}(\boldsymbol{z}, \boldsymbol{w})=\frac{1}{n !} \operatorname{det}\left(\left(\left(\left(1-z_{j} \bar{w}_{k}\right)^{-\lambda}\right)\right)_{j, k=1}^{n}\right), \boldsymbol{z}, \boldsymbol{w} \in \mathbb{D}^{n} .
$$

Proof. For $\boldsymbol{z}, \boldsymbol{w}$ in $\mathbb{D}^{n}$, we have

$$
\begin{aligned}
\sum_{\boldsymbol{p} \geq 0} e_{\boldsymbol{p}}(\boldsymbol{z}) \overline{e_{\boldsymbol{p}}(\boldsymbol{w})} & =\frac{1}{n !} \sum_{\boldsymbol{p} \geq 0} \frac{(\lambda)_{\boldsymbol{p}}}{\boldsymbol{p} !} \operatorname{det}\left(\left(\left(z_{k}^{p_{j}}\right)\right)_{j, k=1}^{n}\right) \operatorname{det}\left(\left(\left(\bar{w}_{k}^{p_{j}}\right)\right)_{j, k=1}^{n}\right) \\
& =\frac{1}{n !} \sum_{\boldsymbol{p} \geq 0} \frac{(\lambda)_{\boldsymbol{p}}}{\boldsymbol{p} !}\left(\sum_{\sigma \in \Sigma_{n}} \operatorname{sgn}(\sigma) \prod_{i=1}^{n} z_{i}^{p_{\sigma(i)}}\right)\left(\sum_{\nu \in \Sigma_{n}} \operatorname{sgn}(\nu) \prod_{i=1}^{n} \bar{w}_{\nu(i)}^{p_{i}}\right) \\
& =\frac{1}{n !} \sum_{\boldsymbol{p} \geq 0} \frac{(\lambda)_{\boldsymbol{p}}}{\boldsymbol{p} !} \sum_{\sigma, \nu \in \Sigma_{n}} \operatorname{sgn}(\sigma) \operatorname{sgn}(\nu) \prod_{i=1}^{n}\left(z_{i} \bar{w}_{\nu \sigma(i)}\right)^{p_{\sigma(i)}} \\
& =\frac{1}{n !} \sum_{\sigma, \nu \in \Sigma_{n}} \operatorname{sgn}(\nu \sigma) \sum_{\boldsymbol{p} \geq 0} \frac{(\lambda)_{\boldsymbol{p}}}{\boldsymbol{p} !} \prod_{i=1}^{n}\left(z_{i} \bar{w}_{\nu \sigma(i)}\right)^{p_{\sigma(i)}} \\
& =\frac{1}{n !} \sum_{\sigma, \nu \in \Sigma_{n}} \operatorname{sgn}(\nu \sigma) \prod_{i=1}^{n}\left(1-z_{i} \bar{w}_{\nu \sigma(i)}\right)^{-\lambda} \\
& =\frac{1}{n !} \sum_{\psi \in \Sigma_{n}} \operatorname{sgn}(\psi) \sum_{\nu \sigma=\psi} \prod_{i=1}^{n}\left(1-z_{i} \bar{w}_{\nu \sigma(i)}\right)^{-\lambda} \\
& =\sum_{\psi, \nu \in \Sigma_{n}} \operatorname{sgn}(\psi) \prod_{i=1}^{n}\left(1-z_{i} \bar{w}_{\psi(i)}\right)^{-\lambda} \\
& =\operatorname{det}\left(\left(\left(\left(1-z_{j} \bar{w}_{k}\right)^{-\lambda}\right)\right)_{j, k=1}^{n}\right) .
\end{aligned}
$$

The desired equality follows from (2.1). 
2.2. Schur function. The determinant function $a_{\boldsymbol{m}+\boldsymbol{\delta}}$ is divisible by each of the differences $z_{i}-z_{j}, 1 \leq i<j \leq n$ and hence by the product

$$
\prod_{1 \leq i<j \leq n}\left(z_{i}-z_{j}\right)=\operatorname{det}\left(\left(\left(z_{i}^{n-j}\right)\right)_{i, j=1}^{n}\right)=a_{\boldsymbol{\delta}}(\boldsymbol{z}) .
$$

The quotient $S_{\boldsymbol{p}}:=a_{\boldsymbol{m}+\boldsymbol{\delta}} / a_{\boldsymbol{\delta}}, \boldsymbol{p}=\boldsymbol{m}+\boldsymbol{\delta}$, is therefore well-defined and is called the Schur function [8, p. 40]. The Schur function $S_{p}$ is symmetric and defines a function on the symmetrized polydisc $\mathbb{G}_{n}$. Since the Jacobian of the map $\mathbf{s}$ : $\mathbb{D}^{n} \rightarrow \mathbb{G}_{n}$ coincides with $a_{\boldsymbol{\delta}}$, it follows from Lemma 2.1 that the Schur functions $\left\{S_{\boldsymbol{p}}:=a_{\boldsymbol{m}+\boldsymbol{\delta}} / a_{\boldsymbol{\delta}}: \boldsymbol{p} \in \llbracket n \rrbracket\right\}$ form a set of mutually orthogonal vectors in $\mathbb{A}^{(\lambda)}\left(\mathbb{G}_{n}\right)$. The linear span of these vectors is dense in $\mathbb{A}^{(\lambda)}\left(\mathbb{G}_{n}\right)$. Also, the norms of these vectors coincide with those of $a_{\boldsymbol{p}}$ in $\mathbb{A}^{(\lambda)}\left(\mathbb{G}_{n}\right)$, modulo the normalizing constant $\left\|J_{\mathbf{s}}\right\|_{\lambda}$, via the unitary map $\Gamma$. Hence $\left\|S_{\boldsymbol{p}}\right\|=\sqrt{\frac{n ! p !}{\left\|J_{\mathbf{s}}\right\|_{\lambda}(\lambda)_{\boldsymbol{p}}}}, \boldsymbol{p} \in \llbracket n \rrbracket$. The set $\left\{\hat{e}_{\boldsymbol{p}}=\right.$ $\left.c_{\boldsymbol{p}} S_{\boldsymbol{p}}: \boldsymbol{p} \in \llbracket n \rrbracket\right\}$ is an orthonormal basis for $\mathbb{A}^{(\lambda)}\left(\mathbb{G}_{n}\right)$, where $c_{\boldsymbol{p}}=\sqrt{\frac{\left\|J_{\mathbf{s}}\right\|_{\lambda}(\lambda)_{\boldsymbol{p}}}{n ! \boldsymbol{p} !}}$. Thus we have proved:

Theorem 2.3. For $\lambda>1$, the reproducing kernel $\mathbf{B}_{\mathbb{G}_{n}}^{(\lambda)}$ for the weighted Bergman space $\mathbb{A}^{(\lambda)}\left(\mathbb{G}_{n}\right)$ on the symmetrized polydisc is given by the formula:

$$
\begin{aligned}
\mathbf{B}_{\mathbb{G}_{n}}^{(\lambda)}(\mathbf{s}(\boldsymbol{z}), \mathbf{s}(\boldsymbol{w})) & =\sum_{\boldsymbol{p} \in \llbracket n \rrbracket} c_{\boldsymbol{p}}^{2} S_{\boldsymbol{p}}(\boldsymbol{z}) \overline{S_{\boldsymbol{p}}(\boldsymbol{w})} \\
& =\frac{\left\|J_{\mathbf{s}}\right\|_{\lambda}^{2}}{n !} \frac{\operatorname{det}\left(\left(\left(\left(1-z_{j} \bar{w}_{k}\right)^{-\lambda}\right)\right)_{j, k=1}^{n}\right)}{a_{\boldsymbol{\delta}}(\boldsymbol{z}) \overline{a_{\boldsymbol{\delta}}(\boldsymbol{w})}}
\end{aligned}
$$

for $\boldsymbol{z}, \boldsymbol{w}$ in $\mathbb{D}^{n}$.

The case $\lambda=2$ corresponds to the Bergman space on the symmetrized polydisc. In this case, $\left\|J_{\mathbf{s}}\right\|_{2}=1$ and the formula for the Bergman kernel, except for the constant factor $\frac{1}{n !}$, was found in [7]. (The factor $\frac{1}{n !}$ appears in our formula because we have chosen the normalization $\|1\|=1$ for the constant function 1 in the Hilbert space $\mathbb{A}^{(\lambda)}\left(\mathbb{G}_{n}\right)$. However, as we will see below, it disappears for the Hardy space on the symmetrized polydisc $\mathbb{G}_{n}$.) However, the methods of this paper are very different from that of [7, and we hope it sheds some light on the nature of these kernel functions.

Corollary 2.4. The Bergman kernel on the symmetrized bidisc in $\mathbb{C}^{2}$ is given by the formula

$$
\mathbf{B}_{\mathbb{G}_{2}}^{(2)}(\boldsymbol{u}, \boldsymbol{v})=\frac{1}{2} \frac{2\left(1+u_{2} \bar{v}_{2}\right)-u_{1} \bar{v}_{1}}{\left(\left(1-u_{2} \bar{v}_{2}\right)^{2}-\left(u_{1}-u_{2} \bar{v}_{1}\right)\left(\bar{v}_{1}-\bar{v}_{2} u_{1}\right)\right)^{2}},
$$

$\boldsymbol{u}=\left(u_{1}, u_{2}\right), \boldsymbol{v}=\left(v_{1}, v_{2}\right) \in \mathbb{G}_{2}$.

This corollary gives an explicit formula for the Bergman kernel function for the symmetrized polydisc which is independent of the symmetrization map $\mathbf{s}$. It is possible to write down similar formulae for $n>2$ using the Jacob-Trudy identity [6. p. 455]. 


\section{The Hardy SPace And the Szegö Kernel FOR THE SYMMETRIZED POLYDISC}

Let $d \Theta$ be the normalized Lebesgue measure on the torus $\mathbb{T}^{n}$, where $\mathbb{T}=\{\alpha$ : $|\alpha|=1\}$ is the unit circle. Let $d \Theta_{\mathbf{s}}$ be the measure supported on the boundary of the symmetrized polydisc $\mathbb{G}_{n}$ obtained by the change of variable formula

$$
\int_{\partial \mathbb{G}_{n}} f d \Theta_{\mathbf{s}}=\int_{\mathbb{T}^{n}}(f \circ \mathbf{s})\left|J_{\mathbf{s}}\right|^{2} d \Theta,
$$

where, as before, $J_{\mathbf{s}}(\boldsymbol{z})$ is the complex Jacobian of the symmetrization map s. Let us define the Hardy space $H^{2}\left(\mathbb{G}_{n}\right)$ on the symmetrized polydisc $\mathbb{G}_{n}$ to be the Hilbert space consisting of those holomorphic functions on $\mathbb{G}_{n}$ for which

$$
\sup _{0<r<1} \int_{\mathbb{T}^{n}}\left|f \circ \mathbf{s}\left(r e^{i \Theta}\right)\right|^{2}\left|J_{\mathbf{s}}\left(r e^{i \Theta}\right)\right|^{2} d \Theta<\infty, e^{i \Theta} \in \mathbb{T}^{n} .
$$

We set the norm of $f \in H^{2}\left(\mathbb{G}_{n}\right)$ to be

$$
\|f\|=\left\|J_{\boldsymbol{s}}\right\|^{-1}\left\{\sup _{0<r<1} \int_{\mathbb{T}^{n}}\left|f \circ \mathbf{s}\left(r e^{i \Theta}\right)\right|^{2}\left|J_{\mathbf{s}}\left(r e^{i \Theta}\right)\right|^{2} d \Theta\right\}^{1 / 2},
$$

where $\left\|J_{\boldsymbol{s}}\right\|^{2}=\int_{\mathbb{T}^{n}}\left|J_{\mathbf{s}}\right|^{2} d \Theta$. This ensures, as before, $\|1\|=1$. Let $H^{2}\left(\mathbb{D}^{n}\right)$ be the Hardy space on the polydisc $\mathbb{D}^{n}$. The operator $\Gamma: H^{2}\left(\mathbb{G}_{n}\right) \longrightarrow H^{2}\left(\mathbb{D}^{n}\right)$ given by $\Gamma(f)=\left\|J_{\boldsymbol{s}}\right\|^{-1} J_{\mathbf{s}}(f \circ \mathbf{s})$ for $f \in H^{2}\left(\mathbb{G}_{n}\right)$ is then easily seen to be an isometry. The subspace of anti-symmetric functions $H_{\text {anti }}^{2}\left(\mathbb{D}^{n}\right)$ in the Hardy space $H^{2}\left(\mathbb{D}^{n}\right)$ coincides with the image of $H^{2}\left(\mathbb{G}_{n}\right)$ under the isometry $\Gamma$. Thus the operator $\Gamma: H^{2}\left(\mathbb{G}_{n}\right) \longrightarrow H_{\text {anti }}^{2}\left(\mathbb{D}^{n}\right)$ is onto and therefore unitary.

Following arguments identical to the one given after Lemma 2.1, we see that the functions $a_{\boldsymbol{p}}, \boldsymbol{p} \in \llbracket n \rrbracket$ continue to be an orthogonal spanning set for the subspace $H_{\text {anti }}^{2}\left(\mathbb{D}^{n}\right)$. All of the vectors $a_{\boldsymbol{p}}$ have the same norm, namely, $\sqrt{n !}$. Consequently, the set of vectors $\left\{e_{\boldsymbol{p}}(\boldsymbol{z}):=\frac{1}{\sqrt{n !}} a_{\boldsymbol{p}}(\boldsymbol{z}): \boldsymbol{p} \in \llbracket n \rrbracket\right\}$ is an orthonormal basis for the subspace $H_{\text {anti }}^{2}\left(\mathbb{D}^{n}\right)$ of the Hardy space on the polydisc, while the set $\left\{\hat{e}_{\boldsymbol{p}}:=\right.$ $\left.\frac{\left\|J_{s}\right\|}{\sqrt{n !}} S_{\boldsymbol{p}}: \boldsymbol{p} \in \llbracket n \rrbracket\right\}$ forms an orthonormal basis for the Hardy space $H^{2}\left(\mathbb{G}_{n}\right)$ of the symmetrized polydisc $\mathbb{G}_{n}$ via the unitary map $\Gamma$. However, $\left\|J_{s}\right\|=\sqrt{n !}$ and consequently, $\hat{e}_{\boldsymbol{p}}=S_{\boldsymbol{p}}$. Thus computations similar to the case $\lambda>1$ yield an explicit formula for the reproducing kernel $K_{\text {anti }}^{(1)}(\boldsymbol{z}, \boldsymbol{w})$ of the subspace $H_{\text {anti }}^{2}\left(\mathbb{D}^{n}\right)$. Indeed,

$$
K_{\text {anti }}^{(1)}(\boldsymbol{z}, \boldsymbol{w})=\frac{1}{n !} \operatorname{det}\left(\left(\left(\left(1-z_{j} \bar{w}_{k}\right)^{-1}\right)\right)_{j, k=1}^{n}\right) .
$$

This is the limiting case, as $\lambda \rightarrow 1$.

Let $\mathbb{S}_{\mathbb{G}_{n}}$ be the reproducing kernel for the Hardy space $H^{2}\left(\mathbb{G}_{n}\right)$. It is natural to call it the Szegö kernel for the symmetrized polydisc $\mathbb{G}_{n}$. Clearly,

$$
\mathbb{S}_{\mathbb{G}_{n}}(\mathbf{s}(\boldsymbol{z}), \mathbf{s}(\boldsymbol{w}))=\frac{\operatorname{det}\left(\left(\left(\left(1-z_{j} \bar{w}_{k}\right)^{-1}\right)\right)_{j, k=1}^{n}\right)}{J_{\mathbf{s}}(\boldsymbol{z}) \overline{J_{\mathbf{s}}(\boldsymbol{w})}}, \boldsymbol{z}, \boldsymbol{w} \in \mathbb{D}^{n}
$$

Now, using the well-known identity due to Cauchy [8, (4.3), p. 63], we have

$$
\mathbb{S}_{\mathbb{G}_{n}}(\mathbf{s}(\boldsymbol{z}), \mathbf{s}(\boldsymbol{w}))=\sum_{\boldsymbol{p} \in \llbracket n \rrbracket} S_{\boldsymbol{p}}(\boldsymbol{z}) \overline{S_{\boldsymbol{p}}(\boldsymbol{w})}=\prod_{j, k=1}^{n}\left(1-z_{j} \bar{w}_{k}\right)^{-1}, \boldsymbol{z}, \boldsymbol{w} \in \mathbb{D}^{n} .
$$


Therefore, we have a formula for the Szegö kernel of the symmetrized polydisc $\mathbb{G}_{n}$, which we separately record below.

Theorem 3.1. The Szegö kernel $\mathbb{S}_{\mathbb{G}_{n}}$ of the symmetrized polydisc $\mathbb{G}_{n}$ is given by the formula

$$
\mathbb{S}_{\mathbb{G}_{n}}(\mathbf{s}(\boldsymbol{z}), \mathbf{s}(\boldsymbol{w}))=\prod_{j, k=1}^{n}\left(1-z_{j} \bar{w}_{k}\right)^{-1}, \boldsymbol{z}, \boldsymbol{w} \in \mathbb{D}^{n} .
$$

Remark 3.2. For simply connected planar domains, the Bergman kernel is a power of the Szegö kernel. As shown in [1, the symmetrized bidisc is a star-like domain and therefore it is simply connected. However, from the explicit computation of these kernels given above, it follows that the Bergman kernel $\mathbf{B}_{\mathbb{G}_{2}}^{(2)}$ is not a power of the Szegö kernel $\mathbb{S}_{\mathbb{G}_{2}}$. As far as we can tell, the symmetrized bidisc $\mathbb{G}_{2}$ seems to be the first example of a simply-connected domain for which the Bergman kernel is not a power of the Szegö kernel.

We point out that the kernel $\mathbb{S}_{\mathbb{G}_{2}}$ has appeared in a somewhat different context earlier [4, p. 2257].

\section{An alternative approach to the computation OF THE KERNEL FUNCTION}

Recall that the weighted Bergman space $\mathbb{A}^{(\lambda)}\left(\mathbb{D}^{n}\right)$ on the polydisc $\mathbb{D}^{n}$ is the $n$-fold tensor product $\bigotimes_{i=1}^{n} \mathbb{A}^{(\lambda)}(\mathbb{D})$ of the weighted Bergman spaces $\mathbb{A}^{(\lambda)}(\mathbb{D})$ on the unit disc $\mathbb{D}$. The equivalence class $\widehat{\Sigma}_{n}$ of finite-dimensional irreducible representations of the permutation group $\Sigma_{n}$ on $n$ symbols is parametrized by the partitions $\boldsymbol{p} \in[n]$. Let $\left(V_{\boldsymbol{p}}, \boldsymbol{p}\right)$ be a representation corresponding to the partition $\boldsymbol{p}$. Then we have the decomposition

$$
\mathbb{A}^{(\lambda)}\left(\mathbb{D}^{n}\right)=\bigoplus_{\boldsymbol{p} \in[n]} \mathbb{A}^{(\lambda)}\left(\mathbb{D}^{n}, \boldsymbol{p}\right)
$$

where

$$
\mathbb{A}^{(\lambda)}\left(\mathbb{D}^{n}, \boldsymbol{p}\right)=\left\{f \in \mathbb{A}^{(\lambda)}\left(\mathbb{D}^{n}, V_{\boldsymbol{p}}\right): \tau(\boldsymbol{s}) f\left(\boldsymbol{s}^{-1} \cdot \boldsymbol{z}\right)=f(\boldsymbol{z}), \boldsymbol{s} \in \Sigma_{n}\right\}
$$

and $\mathbb{A}^{(\lambda)}\left(\mathbb{D}^{n}, \boldsymbol{p}\right) \cong \mathbb{A}^{(\lambda)}\left(\mathbb{D}, V_{\boldsymbol{p}}^{\prime}\right) \otimes V_{\boldsymbol{p}}^{\prime}$. The orthogonal projection $\mathbb{P}_{\boldsymbol{p}}: \mathbb{A}^{(\lambda)}\left(\mathbb{D}^{n}\right) \rightarrow$ $\mathbb{A}^{(\lambda)}\left(\mathbb{D}^{n}, \boldsymbol{p}\right)$ is given by the formula

$$
\left(\mathbb{P}_{\boldsymbol{p}} f\right)(\boldsymbol{z})=\frac{\chi_{\boldsymbol{p}}(1)}{n !} \sum_{\tau} \chi_{\boldsymbol{p}}(\tau) f\left(\tau^{-1} \cdot \boldsymbol{z}\right)
$$

where the sum is over all $\tau$ in $\Sigma_{n}$ and $\chi_{p}$ is the character corresponding to the representation $V_{\boldsymbol{p}}$. Schur orthogonality relations ensure that $\mathbb{P}_{\boldsymbol{p}}^{2}=\mathbb{P}_{\boldsymbol{p}}$, and it follows that $\mathbb{P}_{\boldsymbol{p}}$ is a projection. Let $V_{\mathrm{sgn}}$ be the sign representation of the permutation group $\Sigma_{n}$ and $\mathbb{P}_{\text {sgn }}$ be the corresponding projection.

Theorem 4.1. The reproducing kernel $K_{\mathrm{sgn}}^{(\lambda)}$ of the Hilbert space $\mathbb{A}^{(\lambda)}\left(\mathbb{D}^{n}, \operatorname{sgn}\right)$ is given by the formula

$$
\begin{aligned}
K_{\mathrm{sgn}}^{(\lambda)}(\boldsymbol{z}, \boldsymbol{w}) & =\left(\mathbb{P}_{\mathrm{sgn}} \otimes \mathbb{P}_{\mathrm{sgn}}^{*}\right)\left(\prod_{i=1}^{n}\left(1-z_{i} \bar{w}_{i}\right)^{-\lambda}\right) \\
& =\frac{a_{\boldsymbol{\delta}}(\boldsymbol{z}) \overline{a_{\boldsymbol{\delta}}(\boldsymbol{w})}}{n !} \sum_{\boldsymbol{p} \in \llbracket n \rrbracket} \frac{(\lambda)_{\boldsymbol{m}+\boldsymbol{\delta}}}{(\boldsymbol{m}+\boldsymbol{\delta}) !} S_{\boldsymbol{p}}(z) \overline{S_{\boldsymbol{p}}(w)}
\end{aligned}
$$


where $S_{\boldsymbol{p}}$ is the Schur function with $\boldsymbol{p}=\boldsymbol{m}+\boldsymbol{\delta}$.

Proof. Recall that $K^{(\lambda)}(\boldsymbol{z}, \boldsymbol{w})=\sum_{\boldsymbol{m} \geq 0} \frac{(\lambda)_{\boldsymbol{m}}}{\boldsymbol{m} !}(\boldsymbol{z} \overline{\boldsymbol{w}})^{\boldsymbol{m}}, \lambda>1$, is the reproducing kernel of the weighted Bergman spaces $\mathbb{A}^{(\lambda)}\left(\mathbb{D}^{n}\right)$. Therefore, we have

$$
\left(\mathbb{P}_{\text {sgn }} \otimes I\right) K_{\boldsymbol{w}}^{(\lambda)}(\boldsymbol{z})=\sum_{\boldsymbol{m} \geq 0} \frac{(\lambda)_{\boldsymbol{m}}}{\boldsymbol{m} !} \overline{\boldsymbol{w}}^{\boldsymbol{m}} \mathbb{P}_{\mathrm{sgn}}\left(\boldsymbol{z}^{\boldsymbol{m}}\right) .
$$

However, $\mathbb{P}_{\mathrm{sgn}}\left(\boldsymbol{z}^{\boldsymbol{m}}\right)=\frac{1}{n !} \operatorname{det}\left(\left(\left(z_{i}^{m_{j}}\right)\right)\right)$, which is zero unless $\boldsymbol{m}$ is in the orbit under $\Sigma_{n}$ of the weight $\boldsymbol{p}$ in $\llbracket n \rrbracket$. So, we conclude that

$$
\begin{aligned}
\left(\mathbb{P}_{\mathrm{sgn}} \otimes \mathbb{P}_{\mathrm{sgn}}^{*}\right) K^{(\lambda)}(\boldsymbol{z}, \boldsymbol{w}) & =\sum_{\boldsymbol{m} \geq 0} \frac{(\lambda)_{\boldsymbol{m}}}{\boldsymbol{m} !} \mathbb{P}_{\mathrm{sgn}}\left(\boldsymbol{z}^{\boldsymbol{m}}\right) \mathbb{P}_{\mathrm{sgn}}\left(\overline{\boldsymbol{w}}^{\boldsymbol{m}}\right) \\
& =\sum_{\boldsymbol{p} \in \llbracket n \rrbracket} \gamma_{\boldsymbol{p}} \frac{(\lambda)_{\boldsymbol{p}}}{\boldsymbol{p} !} a_{\boldsymbol{p}}(\boldsymbol{z}) \overline{a_{\boldsymbol{p}}(\boldsymbol{w})} \\
& =a_{\boldsymbol{\delta}}(\boldsymbol{z}) \overline{a_{\boldsymbol{\delta}}(\boldsymbol{w})} \sum_{\boldsymbol{p} \in \llbracket n \rrbracket} \gamma_{\boldsymbol{p}} \frac{(\lambda)_{\boldsymbol{p}}}{(\boldsymbol{p}) !} S_{\boldsymbol{p}}(\boldsymbol{z}) \overline{S_{\boldsymbol{p}}(\boldsymbol{w})}
\end{aligned}
$$

It is easy to see that $\gamma_{\boldsymbol{p}}=\frac{1}{n !}, p \in \llbracket n \rrbracket$, completing the proof.

Clearly, the two kernel functions $K_{\mathrm{sgn}}^{(\lambda)}$ and $K_{\text {anti }}^{(\lambda)}$ are equal. As before, the kernel function $K_{\mathrm{sgn}}^{(\lambda)}$, via the unitary map $\Gamma$, gives a kernel function for the weighted Bergman spaces $\mathbb{A}^{(\lambda)}\left(\mathbb{G}_{n}\right)$ on the symmetrized polydisc $\mathbb{G}_{n}$. Furthermore, if $\lambda=1$, then

$$
\begin{aligned}
\mathbb{S}_{\mathbb{G}_{n}}(\boldsymbol{s}(\boldsymbol{z}), \boldsymbol{s}(\boldsymbol{w})) & =\frac{n !}{a_{\boldsymbol{\delta}}(\boldsymbol{z}) \overline{a_{\boldsymbol{\delta}}(\boldsymbol{w})}} K_{\mathrm{sgn}}^{(1)}(\boldsymbol{z}, \boldsymbol{w}) \\
& =\sum_{\boldsymbol{p} \in \llbracket n \rrbracket} S_{\boldsymbol{p}}(\boldsymbol{z}) \overline{S_{\boldsymbol{p}}(\boldsymbol{w})} \\
& =\prod_{i, j=1}^{n}\left(1-z_{i} \bar{w}_{j}\right)^{-1}, \boldsymbol{z}, \boldsymbol{w} \in \mathbb{D}^{n},
\end{aligned}
$$

where the last equality is the formula [8, (4.3), p. 63].

\section{ACKNOWLEDGEMENT}

The authors would like to thank the anonymous referee for several remarks which helped in clarifying a number of obscure arguments.

\section{REFERENCES}

1. J. Agler and N. J. Young, The hyperbolic geometry of the symmetrized bidisc, J. Geom. Anal., 14 (2004), 375-403. MR2077158 (2005e:32022)

2. E. Bedford, Proper holomorphic mappings, Bull. Amer. Math. Soc. (New Series), 10 (1984), 157-175. MR0733691 (85b:32041)

3. S. R. Bell, The Bergman kernel function and proper holomorphic mappings, Trans. Amer. Math. Soc., 270 (1982), 685-691. MR645338 (83i:32033)

4. R. G. Douglas and G. Misra, Equivalence of quotient Hilbert modules. II, Trans. Amer. Math. Soc., 360 (2008), 2229-2264. MR2366981 (2009c:47024)

5. R. G. Douglas and V. I. Paulsen, Hilbert modules over function algebras, Pitman Research Notes in Mathematics Series, 217, Longman Scientific and Technical, Harlow, 1989. MR.1028546 (91g:46084) 
6. W. Fulton and J. Harris, Representation theory, a first course, Springer-Verlag, New York, 1991. MR 1153249(93a:20069)

7. A. Edigarian and W. Zwonek, Geometry of the symmetrized polydisc, Arch. Math., 84 (2005), 364-374. MR2135687 (2006b:32020)

8. I. G. Macdonald, Symmetric functions and Hall polynomials, Oxford University Press, New York, 1995. MR.1354144 (96h:05207)

9. W. Rudin, Proper holomorphic maps and finite reflection groups, Indiana Univ. Math. J., 31 (1982), 701-720. MR667790 (84d:32038)

Department of Mathematics, Indian Institute of Science, Bangalore 560 012, India

E-mail address: gm@math.iisc.ernet.in

Indian Institute of Science Education and Research, Kolkata, Mohanpur Campus, Mohanpur (West Bengal) 741 252, India

E-mail address: ssroy@iiserkol.ac.in

Department of Mathematics, Chalmers University of Technology and Gothenburg University, S-412 96 Gothenburg, SwEden

E-mail address: genkai@chalmers.se 